\title{
30 Jahre Pflegeversicherung: Ein denkwürdiges Jubiläum
}

VOLKER LEIENBACH, ANDREAS BESCHE

Dr. Volker Leienbach ist Verbandsdirektor des Verbandes der Privaten Krankenversicherung (PKV) in Köln und Berlin

Andreas Besche ist Leiter des Bereichs Pflege im Verband der Privaten Krankenversicherung (PKV) in Köln

30 Jahre alt sein und von allen für 20 gehalten zu werden - was für die einen das größte Kompliment wäre, ist in der Pflegeversicherung eine sozialpolitische Gedächtnislücke. Denn der Deutsche Bundestag hat zwar vor 20 Jahren die Einführung der Sozialen Pflegeversicherung (SPV) und damit die Etablierung eines völlig neuen Zweiges der Sozialversicherung im Sozialgesetzbuch beschlossen. Tatsächlich gab es zu diesem Zeitpunkt aber schon seit einem Jahrzehnt eine Absicherung gegen das Pflegerisiko: durch die Private Krankenversicherung.

\section{Die „Erfindung“ der Pflegeversicherung}

\subsection{Pflegebedürftigkeit als gesellschaftliches Phänomen}

Seit Gründung des Deutschen Reiches im Jahr 1871 bewegt sich die Lebenserwartung der Bevölkerung stetig nach oben. Lag die Lebenserwartung für im Jahr 1871 geborene Mädchen (Jungen) noch bei durchschnittlich 38,5 Jahren (35,6 Jahren), ${ }^{1}$ wird die prognostizierte Lebenserwartung für im Jahr 2030 geborene Mädchen (Jungen) voraussichtlich schon bei 85,4 Jahren (81,2 Jahren) liegen. ${ }^{2}$ Mit der stetig steigenden Lebenserwartung entstand das Phänomen der Pflegebedürftigkeit. Spätestens in den 70er Jahren des letzen Jahrhunderts galt Pflegebedürftigkeit - neben dem Krankheitsrisiko - als neues Lebensrisiko im Alter. Denn die Kosten der Pflegebedürftigkeit konnten zumeist nicht von den pflegebedürftigen Personen selbst getragen werden. Vielmehr mussten die Betroffenen in aller Regel Leistungen der Sozialhilfe oder andere staatliche Unterstützungen in Anspruch nehmen. Die finanziellen „Lasten“ der Kommunen nahmen infolgedessen kontinuierlich zu.

Schon ein Gutachten des Kuratoriums Deutsche Altershilfe aus dem Jahre 1974 hat auf die (neuen) gesellschaftlichen $\mathrm{Ri}$ siken und Umstände der Pflegebedürftigkeit aufmerksam gemacht. ${ }^{3}$ Die Reaktionen in der privaten Versicherungswirtschaft ließen nicht lange auf sich warten. Für einen sehr eingeschränkten Personen- und Versichertenkreis führte die Bayerische Beamtenkrankenkasse Mitte 1978 eine Urform der heutigen Pflegeversicherung als Pflegekostentagegeldversicherung ein. ${ }^{4}$ Große Kreise der Bevölkerung konnten von diesem Neuangebot allerdings nicht profitieren. Zugang zu dieser Pflegeversicherung erhielten nur diejenigen Versicherten, die zwingend auch eine Krankheitskostenvollversicherung bei der Bayerischen Beamtenkrankenkasse abgeschlossen hatten und sie dauerhaft (lebenslang) aufrecht erhielten. Zudem war diese Urform der Pflegeversicherung räumlich auf das Bundesland Bayern beschränkt. Von der „Geburtsstunde“ der Pflegeversicherung als neuer Versicherungszweig konnte also in den 70er Jahren noch keine Rede sein.

Aber die Entwicklung war nicht mehr aufzuhalten. Das Problem der (finanziell nicht abgesicherten) Pflegebedürftigkeit nahm stetig zu. 1980 erwähnt der PKVVerband erstmals die denkbare Möglichkeit einer Versicherung zur Absicherung des Pflegerisikos in einer Stellungnahme. ${ }^{5} 1981$ gibt die Konferenz der Ge- 
sundheitsminister der Länder Gutachten zur Finanzierbarkeit des Pflegerisikos in Auftrag. ${ }^{6}$ Anfang 1983 sichert die neue Bundesregierung zu, sich prioritär mit der Finanzierbarkeit der Pflegebedürftigkeit - unter Abstimmung mit den Ländern - zu beschäftigen. ${ }^{7}$ Aber in der Politik gab es vor allem Uneinigkeit über den richtigen Weg. Faktisch konnte man sich auf keine konkreten Maßnahmen einigen. Eine allgemeine Versicherungspflicht in einer Sozialversicherung lehnte die Bundesregierung damals explizi ab. Vor der Mitgliederversammlung des Verbandes der Privaten Krankenversicherung im Jahr 1984 hatte Bundesarbeitsminister Blüm ${ }^{8}$ noch erklärt: „Eine gesetzliche Pflegeversicherung für den Pflegefall kommt nicht in Betracht; eine Pflegeversicherung schafft ibre eigene Nachfrage." Derweil signalisierte die Private Krankenversicherung (PKV) der Politik, einer versicherungstechnischen Lösung positiv gegenüber zu stehen. ${ }^{10}$

\subsection{Das Jahr 1984: Die „Geburts- stunde" der Pflegeversicherung}

Während sich die Spitzenverbände der GKV noch grundsätzlich gegen eine versicherungsrechtliche Absicherung des Pflegerisikos aussprachen und das Pflegerisiko in den bestehenden Strukturen oder durch Steuermittel „auffangen“ wollten, legte 1981 die HALLESCHE Krankenversicherung als erste bundesweit tätige private Krankenversicherung dem Bundesaufsichtsamt für das Versicherungswesen den Entwurf für eine Pflegeversicherung vor. ${ }^{11}$ Wenig später begann der Verband der Privaten Krankenversicherung mit der Ausarbeitung von Musterbedingungen zur Pflegeversicherung (MB/PV). ${ }^{12}$ Derartige Musterbedingungen bildeten die Voraussetzung dafür, dass großen Kreisen der Bevölkerung eine freiwillige Pflegeversicherung als eigene von der Krankenversicherung unabhängige Versicherungslösung zur Abdeckung des Pflegerisikos von einer Vielzahl von Versicherungsunternehmen angeboten werden konnte. Vorbild waren die Musterbedingungen zur Krankenversicherung.

Im Jahr 1984 war es soweit. In Zusammenarbeit mit dem Bundesaufsichtsamt für das Versicherungswesen (BAV) wurden die Musterbedingungen zur Pflegeversicherung (MB/PV) fertiggestellt, von der Mitgliederversammlung des Verbandes der Privaten Krankenversicherung (PKV) am 11. November 1984 verabschiedet und den von den Unternehmen der Privaten Krankenversicherung zu schaffenden Pflegeversicherungstarifen als vertraglicher Rahmen zu Grunde gelegt.

Die Musterbedingungen zur privaten Pflegeversicherung (MB/PV) ermöglichten den privaten Versicherungsunternehmen das Angebot einer kapitalgedeckten Pflegekostenversicherung oder Pflegetagegeldversicherung. Die Pflegekostenversicherung gewährt dem Versicherten bei Eintritt des Leistungsfalles einen Prozentsatz der nachgewiesenen Pflegekosten. Typischer Weise werden bis zu einem Höchstbetrag bis zu $80 \%$ der entstandenen Pflegekosten erstattet. Die Pflegetagegeldversicherung dagegen leistet dem Versicherten eine im Voraus vereinbarte Summe pro Pflegetag. Diese Summe wird unabhängig davon gezahlt, ob Pflegeleistungen stationär, ambulant oder durch pflegende Angehörige erbracht werden.

Die 1984 vorgelegten Musterbedingungen zur Pflegeversicherung (MB/PV) definierten erstmals Merkmale für einen Versicherungsfall bei Pflegebedürftigkeit. Von nun an gab es objektive Kriterien, ab wann und in welchem Maße bei einer versicherten Person Pflegebedürftigkeit vorliegt. Dabei berücksichtigte man insbesondere Ergebnisse einer Studie des National Center of Health Statistics (NCHS). Diese US-amerikanische Behörde hat 1979 und 1980 eine Befragung von insgesamt über 200.000 Personen in den USA durchgeführt. ${ }^{13}$ Im Mittelpunkt der Befragung stand, ob (und in welchem Umfang) Pflegebedürftige (noch) in der Lage waren, bestimmte Handlungen des Alltags ohne oder mit Hilfe von Hilfsmitteln und/oder anderen Personen auszuführen. Als „Activites of Daily Living $(A D L)$ " waren unter anderem folgende Tätigkeiten spezifiziert: Spazierengehen, Verlassen der Wobnung, Benutzung der Toilette, Baden, An- und Auskleiden, Einnahme der Mablzeiten und Aufstehen und zu Bett Gehen. ${ }^{14}$

\subsection{Vorläufer der Pflegestufen und des Pflegebedürftigkeitsbegriffs}

Mit der Vorlage der Musterbedingungen zur Pflegeversicherung (MB/PV) war 1984 auch der Grundstein zu den heutigen Pflegestufen und zum Pflegebedürftigkeitsbegriff in der Pflegepflichtversicherung gelegt. Denn von den Versicherungsgesellschaften wurden die Mus- terbedingungen zur Pflegeversicherung in ihren Tarifbedingungen erheblich konkretisiert. Dabei ist ein neuer Pflegebedürftigkeitsbegriff zu Grunde gelegt worden, der dem der späteren Sozialen Pflichtversicherung in $\$ 14$ Sozialgesetzbuch XI schon sehr ähnlich war. ${ }^{15}$

1 Vgl. Statistisches Bundesamt (2012), Durchschnittliche weitere Lebenserwartung nach Altersstufen ab 1871, unter www.destatis. de/DE/ZahlenFakten/GesellschaftStaat/Bevoelkerung/Sterbefaelle/Tabellen.

2 Vgl. Statistisches Bundesamt (2011), S. 13 f.

3 Kuratorium Deutsche Altershilfe „Gutachten über die stationäre Behandlung von Krankheiten im Alter und über die Kostenübernahme durch die gesetzlichen Krankenkassen“", 1974.

4 Vgl. Verband der Privaten Krankenversicherung (1983), Rechenschaftsbericht, S. 79.

5 Im Rahmen einer Anhörung einer Bund-Länder-Arbeitsgruppe „Aufbau und Finanzierung stationärer und ambulanter Pflegedienste", hat die PKV die Gelegenheit zu einer schriftlichen Stellungnahme erhalten, vgl. dazu Verband der Privaten Krankenversicherung (1980), Rechenschaftsbericht, S. 97.

6 Vgl. Verband der Privaten Krankenversicherung (1981), Rechenschaftsbericht, S. 93.

7 Deutscher Bundestag (1982/1983), Bundestagdrucksachen $9 / 2046$ und 9/2401.

8 Vertreten von Wolfgang Vogt MdB, Parlamentarischer Staatssekretär im Bundesministerium für Arbeit.

9 Vgl. Vortrag, 14. Juni 1984 in Konstanz: „Die soziale Absicherung bei Pflegebedürftigkeit ist nicht befriedigend. Trotzdem sage ich ohne Wenn und Aber: Eine gesetzliche Pflichtversicherung für den Pflegefall kommt nicht in Betracht. Eine Pflegeversicherung schafft ihre eigene Nachfrage. Und nichts wäre unmenschlicher, als alte Menschen aus den Familien in die Pflegeheime abzuschieben, damit sich die Versicherungsbeiträge auch amortisieren. Uns ist ein Bündel kleiner realisierbarer Maßnahmen lieber. Dabei müssen die Möglichkeiten der privaten Vorsorge einen ganz wesentlichen Raum einnehmen."

$10 \mathrm{Vgl}$. Verband der Privaten Krankenversicherung (1983), Rechenschaftsbericht, S. 79.

$11 \mathrm{Vgl}$. Hallesche Krankenversicherung (1984), S. 25; Hallesche Krankenversicherung (1985), S. 15.

12 Vgl. Verband der Privaten Krankenversicherung (1983), Rechenschaftsbericht, S. 79

13 Vgl. Holl, A.; Kakies. P.; Richter, A. (1985), S. $163 \mathrm{f}$.

14 Vgl. § 1 Abs. 3 MB/PV: Als Verrichtung im Ablauf des täglichen Lebens gelten Aufstehen und Zubettgehen, An- und Auskleiden, Waschen, Kämmen und Rasieren, Einnehmen von Mahlzeiten und Getränken, Stuhlgang und Wasserlassen."

15 Im Sozialrecht gab es zu dieser Zeit unterschiedliche Pflegebedürftigkeitsbegriffe z.B. im $§ 35$ Bundesversorgungsgesetz für das soziale Entschädigungsrecht, § 558 RVO für die gesetzliche Unfallversicherung, § 34 Beamtenversorgungsgesetz für die Beamtenversorgung, $\$ \S 68,69$ Bundessozialhilfegesetz für die Sozialhilfe, § 267 Lastenausgleichsgesetz und § 6 der Beihilfevorschriften. Der Gesetzgeber hat auf die Möglichkeit, den Pflegebedürftigkeitsbegriff zu vereinheitlichen, zu diesem Zeitpunkt verzichtet. Vgl. dazu Besche, A. (1995), S. 18 
Um den Gesamtgrad der Einschränkung von Pflegebedürftigen in den Verrichtungen des täglichen Lebens messen und bewerten zu können, sind von den Versicherungsunternehmen neue Bewertungsmaßstäbe eingeführt worden. Es entstand zum Beispiel ein Punktesystem ${ }^{16}$, das je nach Schwere der körperlichen Einschränkungen den Versicherten eine vorher definierte Anzahl von Punkten zuordnete. Je mehr Punkte die Pflegebedürftigen „,erreichten“, desto größer war der prozentuale Anteil, der von der im Pflegefall vereinbarten Versicherungssumme geleistet wurde. Auf diese Weise konnte der Grad der Einschränkung und damit das $\mathrm{Maß}$ der Pflegebedürftigkeit gemessen und andererseits die Höhe der Leistung hierzu in einen direkten Bezug gesetzt werden.

Die Messung eines Hilfebedarfs, der - wie in der heutigen Pflegepflichtversicherung - nach Zeitaufwand berechnet und dann den jeweiligen Pflegestufen zugeordnet wurde, kannte das damalige Punktesystem der Privaten Krankenversicherung (noch) nicht. Gleichwohl dürfte es auf der Hand liegen, dass eine pflegebedürftige Person bei hoher „Punktzahl“ auch einen entsprechend zeitlich höheren Pflegebedarf im System der Pflegepflichtversicherung hatte. Das damalige Punktesystem der PKV kann damit als Vorläufer der Pflegestufen von heute angesehen werden. Unter Zugrundelegung ähnlicher Beurteilungskriterien kam man zu ähnlichen oder vergleichbaren Ergebnissen. ${ }^{1718}$

\section{Die Pflegepflichtversicherung}

\subsection{Kapitaldeckung oder Umlagefinanzierung}

Während im Jahr 1986 bereits 16,19 im Jahr 1988 schon 21 Unternehmen ${ }^{20}$ der Privaten Krankenversicherung kapitalgedeckte private Pflegekostenversicherungen und/oder Pflegetagegeldversicherungen anboten, war eine allgemeine Vorsorgepflicht zur Absicherung des Pflegerisikos noch nicht absehbar. In der Politik stritt man weiterhin über unterschiedliche Lösungsvorschläge. ${ }^{21}$ Während das Bundesland Hessen ${ }^{22}$ in einem Gesetzentwurf aus dem Jahr 1986 schon von der Einführung einer neuen Säule der Sozialversicherung sprach, schlugen andere Bundesländer weiter- hin eine vollständige Steuerfinanzierung vor.

1989 wurde im Land Baden-Württemberg ein „Diskussionsentwurf Finanzielle Absicherung des Pflegerisikos-Einführung einer Vorsorgepflicht" ausgearbeitet und in Abstimmung mit Bayern zu einem Gesetzentwurf weiterentwickelt. Der Diskussionsentwurf sah eine allgemeine (lebenslange) Vorsorgepflicht ab Vollendung des 45. Lebensjahres vor. Es wurde bereits zwischen Leistungsstufen unterschieden. Der Beitrag sollte - bei Berücksichtigung von Kinderfreibeträgen und staatlichen Prämienzuschüssen - geschlechts- und risikounabhängig im Kapitaldeckungsverfahren kalkuliert werden. ${ }^{23}$ Vorbild war das von Beginn an in der Privaten Pflegeversicherung praktizierte Kapitaldeckungsverfahren. ${ }^{24}$

Die Präferenz für das Kapitaldeckungsverfahren hatte gute Gründe. Schon Anfang der 90er Jahre war der überwiegende Teil der Wissenschaft der Meinung, dass sich gerade das Pflegerisiko besonders für das Kapitaldeckungsverfahren eigne. Angesichts der Nachteile der Umlagefinanzierung im demografischen Wandel und angesichts des Tatbestandes, dass das Pflegerisiko noch mehr als das Krankheitsrisiko ein altersabhängiges, im höheren Alter exponentiell ansteigendes Risiko darstellt, wäre eine kapitalgedeckte Pflegepflichtversicherung ordnungspolitisch die beste Lösung gewesen.

Gleichwohl hätte die Etablierung einer kapitalgedeckten Pflegepflichtversicherung einen fiskalischen Preis gehabt. Weil Vorsorge durch Kapitaldeckung zunächst einmal aufgebaut werden muss, hätte die Politik die in einer Übergangsphase zwangsläufig entstehenden sozialen Härten durch einen Steuertransfer finanziell „abfedern“ müssen. Eine Herausforderung, die heute wie damals an Grenzen der öffentlichen Haushalte stößt.

Umlagefinanzierte Vorschläge zur Pflegepflichtversicherung konnten diese steuerpolitischen Belastungen in der Übergangsphase - zu Lasten der nachfolgenden Generationen - vermeiden. Ein Argument, das in der Politik angesichts knapper Haushalte gerade in den Jahren nach der Deutschen Einheit Gewicht hatte. Damit war (für den Großteil der Bevölkerung) der Weg in eine umlagefinanzierte Pflegepflichtversicherung vorgezeichnet.

\subsection{Pflegeversicherung folgt Krankenversicherung}

Ab 1992 nahmen die politischen Bemühungen, zur Absicherung des Pflegerisikos einen weiteren Sozialversicherungszweig zu begründen, stetig zu. 1993 wurde das sogenannte Blüm-Modell vorgelegt. Danach sollte die gesamte Bevölkerung einschließlich der 7 Mio. Privatversicherten im Rahmen einer neuen Säule der Sozialversicherung (SGB XI) in eine umlagefinanzierte Soziale Pflegeversicherung (SPV) einbezogen werden (Bürgerpflegeversicherung).

16 Vgl. u.a. Münchener Verein Krankenversicherung a. G

17 Hilfsregelungen in der GKV, die das Pflegerisiko für „Schwerpflegebedürftige“ ab 1991 zumindest in Teilen in den bestehenden Strukturen der gesetzlichen Krankenversicherung sicherstellen sollten (damalige $\$ \S$ 53 ff. SGB V), bewährten sich in der Praxis nicht. Der Bewertungsmaßstab „schwerpflegebedürftig" führte im Alltag zu Abgrenzungsschwierigkeiten zu den Leistungen der Sozialhilfe. Darüber hinaus hatte der Gesetzgeber auch die Auslegung des unbestimmten Rechtsbegriffs der „Schwerpflegebedürftigkeit" der Selbstverwaltung der Krankenkassen überlassen. Die Folge waren zahlreiche juristische Auseinandersetzungen. Letztlich mussten die Sozialgerichte die Schwelle von „Schwerpflegebedürftigkeit" definieren und festlegen.

18 Viele weitere Ideen der privaten Pflegeversicherungen fanden ihren Niederschlag in den späteren Regelungen der sozialen Pflegepflichtversicherung. Ein Beispiel: Soweit die einzelnen neu geschaffenen Tarife der privaten Pflegeversicherung die Übernahme von Leistungen für häusliche Pflege vorsahen, sollten Zahlungen nur geleistet werden können, wenn die Leistungen von öffentlichen oder freigemeinnützigen Pflege- oder Sozialstationen oder durch staatlich anerkanntes Pflegepersonal erbracht würden, während die Leistung im stationären Bereich daran anknüpfte, dass sie in öffentlichen oder staatlich konzessionierten Einrichtungen erfolgte. Auch diese Voraussetzungen, die einerseits eine gewisse Leistungsqualität gewährleisten aber auch Leistungsmissbrauch verhindern, ähneln sehr dem späteren System der Versorgungsverträge, wie sie als Leistungsvoraussetzung für die SPV in $§ 72$ SGB XI formuliert wurden.

19 Vgl. Verband der Privaten Krankenversicherung (1986), Rechenschaftsbericht, S. 42.

$20 \mathrm{Vgl}$. Verband der Privaten Krankenversicherung (1988), Rechenschaftsbericht, S. 48.

21 Vgl. dazu die Darstellung aller Vorschläge und Entwürfe zwischen 1986 und 1992 bei Jung, K. (1995), S 48 f.

22 Bundesrat (1986), Drucksache 81/86 vom 7.2.1986, „Entwurf eines Gesetzes zur Absicherung des Risikos der Pflegebedürftigkeit (Pflegeversicherungsgesetz)."

23 Vgl. Verband der Privaten Krankenversicherung (1989), Rechenschaftsbericht, S. 98 f.

24 Detaillierte Darstellung bei Verband der Privaten Krankenversicherung (1989), Rechenschaftsbericht, S. 98. 
Das letztendlich am 26. Mai 1994 vom Deutschen Bundestag verabschiedete und zum 1. Januar 1995 in Kraft getretene Pflegeversicherungsgesetz orientierte sich dagegen - abweichend vom Blüm-Modell - am Grundsatz „Pflegeversicherung folgt Krankenversicherung". Damit war auch die PKV Träger der Pflegepflichtversicherung. ${ }^{25}$ Die Vollversicherten der Privaten Krankenversicherung wurden damit ab dem 1.1.1995 automatisch in der privaten Pflegepflichtversicherung (PPV) versichert. ${ }^{26}$

Die PPV funktioniert im Grundsatz analog der freiwilligen Pflegekostenversicherungen und Pflegetagegeldversicherungen nach dem Prinzip der Kapitaldeckung: ${ }^{27}$ Jede Generation von Versicherten sorgt durch die Bildung von Alterungsrückstellungen frühzeitig für ihr mit dem Alter steigendes eigenes Pflegerisiko vor. Durch diese kapitalgedeckte Finanzierung der Pflegeausgaben werden keine Finanzierungslasten auf kommende Beitragszahlergenerationen verschoben. Vielmehr wird ein Kapitalstock zur Zukunftsvorsorge und Entlastung der nachfolgenden Generation aufgebaut. Diese Kapitalbildung zahlt sich in der Zukunft aus: Sie stabilisiert die Beiträge zur Pflegeversicherung im Alter und macht die Privatversicherten von der sich ändernden Altersstruktur der Bevölkerung unabhängiger. ${ }^{28}$

Bei der umlagefinanzierten Sozialen Pflegeversicherung werden die laufenden Pflegekosten dagegen über aktuelle, in die Umlage eingezahlte Beiträge finanziert. Eine Vorsorge findet nicht statt. Die Kosten für den demografisch bedingten Anstieg der Anzahl der Pflegebedürftigen müssen überwiegend von der nächsten Generation getragen werden.

Die Soziale Pflegeversicherung wird als umlagefinanzierte Säule zu erheblichen Mehrbelastungen für die zukünftigen Generationen führen. Denn im Umlageverfahren der SPV müssen die künftigen Versicherungsleistungen von der Generation der dann Erwerbstätigen gezahlt werden. Die alternde Gesellschaft wird diese Finanzierungsweise allerdings auf eine ernste Probe stellen. Laut Statistischem Bundesamt wird die Zahl der über 80-Jährigen im Jahr 2050 dreimal so hoch sein wie heute. Gleichzeitig wird es ein Drittel weniger Menschen im erwerbsfähigen Alter geben. Es steigt also nicht nur der Pflegebedarf - mit dem Rückgang der Erwerbsfähigen schwindet in der SPV auch immer mehr die Refinanzierungsbasis.

\section{Die private Pflegeversicherung im Wandel der Zeit}

\subsection{Pflege als (un)versicherbares Risiko?}

1984 ging die private Pflegeversicherung mit einer hohen Erwartungshaltung seitens der PKV an den Start. Die Nachfrage indes blieb weit hinter diesen Erwartungen zurück. Das Interesse in der Bevölkerung war zurückhaltend. Bis 1986 wurden lediglich 28.000 private Pflegeversicherungen abgeschlossen (vgl. Tabelle 1). Bis zum Jahr 1994 - dem Beginn der Pflegepflichtversicherung - gab es weniger als 320.000 Pflegeversicherungen im Bestand der PKV.

Dabei hatte zunächst vieles dafür gesprochen, dass sich die Private Pflegeversicherung schneller etablieren würde. Das Pflegerisiko war in den 80er Jahren ein neues, versicherungsfähiges und hinreichend großes Lebensrisiko, das bei Eintritt schnell zur persönlichen Überforderung führen kann. Es ließ sich eindeutig vom Grundschutz und vom Leistungskatalog der Gesetzlichen Krankenversicherung (GKV) oder einer anderen Pflichtversicherung abgrenzen. Das Pflegerisiko konnte darüber hinaus versicherungsmathematisch und kalkulatorisch von den Versicherungen gut abgebildet werden. Und: Die Beiträge waren (insbesondere für junge Menschen) moderat bis niedrig.

Ein wesentlicher Grund für das dennoch verhaltene Interesse an der Pflegeversicherung lag wohl - neben dem Ausbleiben einer wiederholt in Aussicht gestellten speziellen steuerlichen Förderung - in der damals in der Gesellschaft noch unterentwickelten Wahrnehmbarkeit des Pflegerisikos. Die Wahrnehmung von Risiken ist unabdingbare Voraussetzung für die Durchsetzung von Versicherungsprodukten am Markt. Im Kontext der Pflegevorsorge spielte auch das Phänomen der Verdrängung eine große Rolle. Insbesondere wenn es sich um Risiken in der fernen Zukunft handelt, ist es nicht unwahrscheinlich, dass Menschen dazu neigen, von einer Nichtbetroffenheit auszugehen. Sie erkennen die Relevanz von Leistungen, die in der Regel am Ende des Lebens benötigt werden, nicht ausreichend an.
Dem Phänomen der Verdrängung des Pflegerisikos kann man prinzipiell mit Aufklärung begegnen. Gerade dazu haben insbesondere zwei öffentliche Diskussionen und gesetzgeberische Initiativen rund um das zunehmend emotional besetzte Thema „Pflegevorsorge“ beigetragen - die Einführung und Etablierung der Pflegepflichtversicherung im Jahr 1994 und der Start der steuerlich geförderten Pflegezusatzversicherung („BahrPflege“) im Jahr 2013.

\subsection{Die (private) Pflegepflichtversicherung}

Die Einführung der Pflegepflichtversicherung hat das Thema „Pflegevorsorge“ stärker im Bewusstsein der Menschen verankert. Erstmals wurde tagesaktuell in einer breiten Öffentlichkeit über das altersabhängige Pflegerisiko informiert, diskutiert und sensibilisiert. Davon hat auch die freiwillige Pflegeversicherung beziehungsweise die von nun an als freiwillige private Zusatzversicherung $\mathrm{zu}$ bezeichnende Pflegeversicherung profitiert. Denn die Pflichtversicherung hat die Pflege(zusatz)versicherung keineswegs verdrängt. Im Gegenteil, die Zahl der Versicherungsverträge im Bereich der Pflege(zusatz)-versicherung hat insbesondere ab Mitte der 90er Jahre eine zumindest verhaltene Dynamik aufgenommen.

25 Vgl. zur Integration der PKV in die Pflegepflichtversicherung, Koch, P.; Uleer, C. (1997), S. 129 f.

26 Mit der Einführung der Pflegepflichtversicherung galt in der PPV - analog zur SPV - ein obligatorischer, modernisierter Pflegebedürftigkeitsbegriff. Ein eigens gegründeter medizinischer Dienst, die MEDICPROOF, übernimmt bis heute die Begutachtung der privatversicherten Antrag steller. Die Gutachten, die von den rund 1.000 freiberuflichen Gutachtern erstellt werden, sind nach der Rechtsprechung des Bundessozialgerichts für die privaten Versicherungsunternehmen verbindlich (BSG Urteile vom 22.8.2001, Az: B 3 P 21/00 und B 3 P 40/01 R). Die MDK-Gutachten für die SPV stellen dagegen lediglich Empfehlungen für die Pflegekassen dar.

27 Zur Funktionsweise der Privaten Pflegepflichtversicherung vgl. Riedel, H. (2003), S. $275 \mathrm{f}$

28 Auch wenn die Kalkulation der privaten Pflegepflichtversicherung grundsätzlich nach dem Kapitaldeckungsverfahren erfolgt, hat die PKV doch einige ihr fremde Elemente akzeptiert. Dazu gehört beispielsweise die beitragsfreie Mitversicherung für Kinder, die Limitierung der Beiträge für Ehegatten oder die Festsetzung eines Höchstbeitrages, der sich am Höchstbeitrag in der Sozialen Pflegepflichtversicherung orientiert. 
Tabelle 1: Versicherte und Versicherungsverträge in der privaten Pflegeversicherung seit 1984

\begin{tabular}{|c|c|c|c|}
\hline Jahr & $\begin{array}{c}\text { Private Pflege- } \\
\text { (zusatz)versicherung* }\end{array}$ & $\begin{array}{l}\text { Private Pflege- } \\
\text { pflichtversicherung' }\end{array}$ & $\begin{array}{l}\text { geförderte private Pflege- } \\
\text { (zusatz)versicherung* }\end{array}$ \\
\hline 1984 & $\begin{array}{l}\text { Vorlage der Musterbe- } \\
\text { dingungen zur privaten } \\
\text { Pflegeversicherung }\end{array}$ & - & - \\
\hline 1985 & $14.300^{* *}$ & - & - \\
\hline 1986 & 28.420 & - & - \\
\hline 1987 & 46.270 & - & - \\
\hline 1988 & 53.700 & - & - \\
\hline 1989 & 76.000 & - & - \\
\hline 1990 & 103.000 & - & - \\
\hline 1991 & 133.000 & - & - \\
\hline 1992 & 195.000 & - & - \\
\hline 1993 & 297.600 & - & - \\
\hline 1994 & 315.900 & - & - \\
\hline 1995 & 379.900 & 7.911 .600 & - \\
\hline 1996 & 402.100 & 7.926 .000 & - \\
\hline 1997 & 412.400 & 8.007 .800 & - \\
\hline 1998 & 543.000 & 8.131 .000 & - \\
\hline 1999 & 570.300 & 8.226 .000 & - \\
\hline 2000 & 605.100 & 8.303 .400 & - \\
\hline 2001 & 655.700 & 8.567 .000 & - \\
\hline 2002 & 690.000 & 8.827 .100 & - \\
\hline 2003 & 749.600 & 8.999 .300 & - \\
\hline 2004 & 787.100 & 9.117 .600 & - \\
\hline 2005 & 832.900 & 9.164 .300 & - \\
\hline 2006 & 988.800 & 9.276 .800 & - \\
\hline 2007 & 1.174 .000 & 9.320 .000 & - \\
\hline 2008 & 1.316 .200 & 9.352 .400 & - \\
\hline 2009 & 1.500 .500 & 9.534 .100 & - \\
\hline 2010 & 1.699 .500 & 9.593 .000 & - \\
\hline 2011 & 1.880 .400 & 9.666 .900 & - \\
\hline 2012 & 2.186 .700 & 9.619 .600 & - \\
\hline 2013 & $2.340 .800^{* * *}$ & $9.538 .600^{* * *}$ & $350.100^{* * * *}$ \\
\hline
\end{tabular}

*Versicherungsverträge; ${ }^{* *}$ geschätzt; ${ }^{* * *}$ Stand $30.11 .2013 ;{ }^{* * * *}$ Stand 31.12 .2013 , plus weitere Verträge mit Laufzeitbeginn nach dem 31.12.2013; ' Versicherte Personen; Quelle: Zahlenberichte des PKV-Verbandes

Bis zur Einführung der Pflegepflichtversicherung im Jahr 1995 sind knapp 380.000 private Versicherungsverträge abgeschlossen worden (vgl. Tabelle 1). Seit 1995 haben sich die Zahlen der bestehenden Versicherungsverträge um den Faktor 6,16 auf 2,34 Mio. Verträge erhöht. ${ }^{29}$ Gleichzeitig stieg die Zahl der Versicherten in der privaten Pflegepflichtversicherung (PPV) von 7,9 Mio. auf fast 9,6 Mio. Menschen.

\subsection{Die geförderte (ergänzende) Pflegezusatzversicherung}

Das Phänomen der Verdrängung des Pflegerisikos funktioniert bis heute. Noch immer machen sich viele Versicherte häufig nicht klar, dass die Pflegepflichtversicherung lediglich eine Art Teilkaskoversicherung darstellt. Vielen pflegebedürftigen Menschen ohne Pflege(zusatz)versicherung sind trotz bestehender Pflegepflichtversicherung nicht in der Lage, für ihre Pflegekosten allein aufzukommen. Ein Beispiel: Die Kosten für eine Unterbringung im Heim bei Pflegestufe III betragen heute im Durchschnitt bei vollstationärer Pflege 3.236,69 Euro pro Monat. Die Pflegepflichtversicherung leistet in dieser Stufe nur 1.550 Euro. Damit bleibt ein Eigenanteil von 1.686,69 Euro, der aus eigener Tasche zu bezahlen ist.

Der Gesetzgeber hat im Jahr 2012 auf diese „Sicherungslücke“ in der Pflege reagiert. Seit dem 1.1.2013 gibt es - neben der klassischen Pflege(zusatz) versicherung und der Pflege-plichtversicherung - als dritte Säule der sozialen Sicherung in der Pflege die staatlich geförderte private Pflegezusatzversicherung („Bahr-Pflege“). Die geförderte Pflegezusatzversicherung bietet allen die Chance, Hilfebedürftigkeit im Pflegefall zu verhindern, die eigene Rente zu schonen und einen Rückgriff auf Zahlungspflichten ihrer Angehörigen zu vermeiden.

Anspruch auf Abschluss der geförderten Pflegezusatzversicherung hat jeder, der in der Pflegepflichtversicherung versichert ist, sofern er das 18. Lebensjahr vollendet hat und bei Vertragsabschluss noch keine Leistungen aus der Pflegepflichtversicherung bezieht. Für die privaten Versicherungsunternehmen gilt Annahmezwang, etwaige Vorerkrankungen haben keinen Einfluss auf das Zustandekommen des Vertrages, den Versicherungsumfang oder die Beitragshöhe. Die Höhe der Beiträge richtet sich nach dem vereinbarten Pflegemonatsgeld und dem Lebensalter des Versicherten bei Vertragsabschluss. Die staatliche Zulage beträgt immer 5 Euro pro Monat. Das entspricht einer Förderquote von bis zu 33 Prozent des Beitrags (5 Euro Zuschuss bei 15 Euro Mindestbeitrag).

Inzwischen boomt die Nachfrage nach geförderten Pflege(zusatz)versicherungen. ${ }^{30}$ Ende Dezember 2013 gab es bereits 350.100 laufende Verträge (vgl. Tabelle 1), dazu kommen weitere Verträge mit

29 Mit in Kraft treten des Pflegeversicherungsgesetzes im Jahr 1995 war auch ein zusätzlicher Sonderausgabenabzugbetrag von jährlich bis zu 360 DM vorgesehen, allerdings nur für Personen ab dem Geburtsjahrgang 1958, also die damals unter 38jährigen. Einige Jahre später ist dieser Sondervorteil durch das Alterseinkünftegesetz jedoch wieder beseitigt worden.

30 Vgl. u.a. Die WELT, 16.11.2013, S. 17; Frankfurter Allgemeine Zeitung (FAZ), 14.11.2013. 
einem später vereinbarten Vertragsbeginn. Damit sind innerhalb von 12 Monaten mehr staatlich geförderte Pflegezusatzversicherungen abgeschlossen worden als im Bereich der klassischen (steuerlich ungeförderten) Pflegeversicherung in den ersten 10 Jahren zwischen 1984 und 1994 zusammen.

Und die Dynamik hält an: Während kurz nach Einführung der (steuerlich) geförderten Pflegezusatzversicherung im Januar etwa 240 neue Verträge pro Arbeitstag abgeschlossen wurden, stieg die Zahl im Juni auf 1.000 Verträge pro Arbeitstag und im Dezember auf 1.600. Angesichts des anhaltenden Kundeninteresses dürfte wohl schon Ende 2014 als ehrgeiziges, aber durchaus realistisches Ziel die Marke von einer Million Verträgen erreicht sein.

\section{Dank der steuerlich geförderten Pflegezusatzversicherung ("Bahr-Pflege") schreitet der Bewusstseinswandel im Bereich der "Pflegevorsorge"
beschleunigt voran.}

Dank der steuerlich geförderten Pflegezusatzversicherung („Bahr-Pflege“) schreitet der Bewusstseinswandel im Bereich der „Pflegevorsorge“ beschleunigt voran. Das lässt sich mit Zahlen unterlegen: Die seit 1984 von der Privaten Krankenversicherung angebotene freiwillige Pflegeversicherung ist von der steuerlich geförderten Pflegezusatzversicherung keinesfalls verdrängt worden; im Gegenteil, seit Beginn der Diskussion um eine steuerlich geförderte Pflegezusatzversicherung im Jahr 2011 hat sich die Zahl der Versicherungsverträge im Bereich der klassischen Pflegezusatzversicherungen von 1,88 Mio. (2011) auf 2,34 Mio. (2013) erhöht (Tabelle 1). Der ZweiJahres-Zuwachs entspricht einem Anstieg von fast 24,5 Prozent (+ 460.000). Damit sind innerhalb von 2 Jahren mehr klassische Pflegezusatzversicherungen abgeschlossen worden als in den ersten 14 Jahren zwischen 1984 und 1997 zusammen.

\subsection{Qualitätsoffensive „Pflege“}

Die 30jährige Geschichte der privaten Pflegeversicherung wird vervollständigt durch Innovationen im Bereich der Pflegequalität. Da die PKV - anders als die Pflegekassen - bundesweit organisiert ist, verfolgt sie stets einen bundeseinheitlichen Ansatz: Das Spektrum reicht von der Pflegeberatung und -begutachtung über die wissenschaftliche Verbesserung der Versorgungspraxis bis hin zur Präventionsarbeit.

Im Jahr 2009 hat die PKV die aufsuchende Pflegeberatung COMPASS gegründet. Bei der „COMPASS Private Pflegeberatung“ erhalten alle Ratsuchenden zunächst unabhängig vom Versicherungsstatus eine kostenlose anonyme Beratung unter der gebührenfreien Service-Nummer 0800 - 1018800. Privatversicherte und ihre Angehörigen können anschließend auf ein flächendeckendes Netz von mobilen Beratern zurückgreifen, um konkrete Hilfe in ihrer eigenen häuslichen Umgebung zu erhalten. Mehrere hunderttausend Beratungen hat die COMPASS $\mathrm{GmbH}$ seit Gründung im Jahr 2009 durchgeführt.

Das Konzept der Sozialen Pflegepflichtversicherung beruhte dagegen auf sogenannten Pflegestützpunkten, die die Betroffenen und ihre Angehörigen bei Bedarf selbst aufsuchen müssen. Das ist nicht nur eine zusätzliche Hürde bei der Bewältigung des Pflegealltags - ein flächendeckendes Netz solcher Stützpunkte gibt es noch immer nicht. Längst hat sich deshalb die aufsuchende Pflegeberatung der „COMPASS Pflegeberatung “ zum Vorbild für das ganze System entwickelt: Im Zuge der letzten Pflegereform hat der Gesetzgeber daher auch für die Pflegekassen eine aufsuchende Beratung zum Standard gemacht.

Auch bei den Qualitätsprüfungen in Pflegeheimen und bei Pflegediensten („Pflege-TÜV“) setzt die Private Krankenversicherung dem Vorgehen der Pflegekassen ein erfolgreiches eigenes Konzept entgegen. Seit 2011 führt der Prüfdienst der PKV entsprechend dem Anteil der Privatversicherten an der Bevölkerung jährlich etwa 2.400 Prüfungen von Pflegeeinrichtungen durch. Anders als die regional tätigen Medizinischen Dienste der Pflegekassen, die bei der Umsetzung der Prüfvorgaben oft je nach Ort erhebliche Unterschiede machen, arbeitet der PKV-Prüfdienst bundesweit nach einheitlichen Vorgaben. Damit macht er den direkten Qualitätsvergleich zwischen Einrichtungen in unterschiedlichen Regionen möglich.

Darüber hinaus verfolgen die Qualitätsprüfer der PKV in den Prüfungen, für die zwei Tage angesetzt werden, einen stark beratungsorientierten Ansatz. Sie kontrollieren die Einrichtungen nicht nur, sondern helfen ihnen dabei ihre Aufgaben besser zu erfüllen. Der Ansatz der PKV geht damit über den reinen Verbraucherschutz hinaus: Die Dualität aus Privater und Gesetzlicher Krankenversicherung führt zu einem positiven Leistungswettbewerb, von dem sowohl Pflegebedürftige als auch Pflegende profitieren.

Um die Pflegequalität auf eine wissenschaftlich fundierte Grundlage zu stellen, hat die PKV zudem im Jahr 2009 die gemeinnützige Stiftung „Zentrum für Qualität in der Pflege“ (ZQP) gegründet. Ziel der Stiftung ist es, Qualität der Pflege und Versorgungspraxis zu verbessern. Das ZQP leitet gezielt wissenschaftliche Untersuchungen zu Fragen ein, bei denen die Fachwelt noch Erkenntnisbedarf hat. Dabei verfolgt es einen multidisziplinären und multiprofessionellen Ansatz und versucht, Wissen aus Theorie und Praxis zu verknüpfen.

Die Projekte zielen dabei auf hohe Praxisrelevanz - und damit auf eine nachhaltige Verbesserung der Versorgung der Pflegebedürftigen. Die Ergebnisse stellt das ZQP der Öffentlichkeit kostenlos zur Verfügung. In Fachkreisen hat es sich inzwischen zu einem nationalen Kompetenzzentrum für den Bereich der Pflegequalität entwickelt.

Seit Januar 2014 engagiert sich die PKV nicht zuletzt auch in der Präventionsarbeit im Bereich der Pflege, um die Lebensqualität im Alter zu erhöhen. In Kooperation mit der Bundeszentrale für gesundheitliche Aufklärung (BZgA) finanziert der PKV-Verband das neue Projekt „Altern in Balance“. Das Präventionsprojekt soll die Selbstbestimmung, Mobilität und Lebensqualität älterer Menschen fördern. Vor allem körperliche Bewegung, geistige Aktivität und soziale Teilhabe scheinen $\mathrm{zu}$ einem gesunden Altern und damit auch zur Vermeidung oder dem Herausschieben von Pflegebedürftigkeit beizutragen. Nach der erfolgreichen Zusammenarbeit in der Aidsprävention sowie in der Prävention von Alkoholmissbrauch durch Kinder und Jugendliche ist dies 
schon die dritte Kooperation der PKV mit der BZgA.

\section{Zusammenfassung und Schlussfolgerung}

Die Pflegeversicherung feiert nicht - wie häufig in der Politik, in den Medien und in der Öffentlichkeit vermutet ihren zwanzigsten, sondern ihren 30. Geburtstag. Denn schon lange vor der Einführung der Pflegepflichtversicherung im Jahr 1994 gab es eine verlässliche Absicherung gegen das Pflegerisiko durch die Private Krankenversicherung. Als „Geburtsstunde“ der Pflegeversicherung kann man das Jahr 1984 betrachten.

30 Jahre Pflegeversicherung stützen sich auf drei tragfähige Säulen: Die 1984 auf den Weg gebrachte freiwillige Pflege(zusatz)versicherung, die 1994 vom Gesetzgeber beschlossene private Pflegepflichtversicherung und die Anfang 2013 etablierte steuerlich geförderte Pflegezusatzversicherung („Bahr-Pflege“). Alle drei Säulen der Pflegeversicherung sind kapitalgedeckt und damit generationengerecht und nachhaltig finanziert.

Einige Fakten zur 30jährigen Geschichte der Pflegeversicherung im Überblick:

- Während im Jahr 1984 Bundesarbeitsminister Blüm ${ }^{31}$ noch erklärte, „eine gesetzliche Pflegeversicherung für den Pflegefall komme nicht in Betracht", hat die PKV mit der Verabschiedung von Musterbedingungen zur Pflegeversicherung (MB/PV) schon im Jahr 1984 die freiwillige Pflegeversicherung als eigene von der Krankenversicherung unabhängige Versicherungslösung zur Abdeckung des Pflegerisikos ins Leben gerufen.

- Die PKV hat mit der neuen Pflegeversicherung schon im Jahr 1984 fortfolgend den Grundstein zu den heutigen Pflegestufen und zum heute gültigen Pflegebedürftigkeitsbegriff in der Pflegepflichtversicherung gelegt.

- Obwohl schon in den 90er Jahren ein großer Teil der Wissenschaft der Meinung war, dass sich das Pflegerisiko besonders für das Kapitaldeckungsverfahren eigne, orientierte sich die 1994 vom Bundestag verabschiedete Pflegepflichtversicherung am Grundsatz „Pflegeversicherung folgt Krankenversicherung“. Demnach wurden „nur“ die Krankenversicherten der PKV in eine kapitalgedeckte Pflegepflichtversicherung integriert.

- Das 1993 zunächst vorgelegte BlümModell zum Einbezug der 7 Mio. Privatversicherten in eine die gesamte Bevölkerung umfassende Soziale Pflegepflichtversicherung (SPV) fand keine Mehrheit. Damit entschied sich der Gesetzgeber im Jahr 1994 bewusst gegen eine umlagefinanzierte Bürger(pflege)versicherung und für den Systemwettbewerb.

- Die Einführung der Pflegepflichtversicherung hat das Thema „Pflegevorsorge" stärker im Bewusstsein der Menschen verankert. Davon hat auch die freiwillige Pflegeversicherung profitiert. Die Zahl der abgeschlossenen Versicherungsverträge im Bereich der Pflege-(zusatz)versicherung hat ab Mitte der 90er Jahre eine neue Dynamik aufgenommen.

- Der Start der „Bahr-Pflege“ hat die klassische Pflegeversicherung nicht verdrängt. Im Gegenteil, seit Beginn der Diskussion um eine geförderte Pflegezusatzversicherung hat sich die Zahl der nicht geförderten Pflegezusatzversicherungen von 1,88 Mio. im Jahr 2011 auf 2,34 Mio. (2013) erhöht. Der Zwei-JahresZuwachs entspricht einem Anstieg von fast $24,5 \%$ (+ 460.000). Damit sind innerhalb von 2 Jahren mehr Pflegezusatzversicherungen abgeschlossen worden als in den ersten 14 Jahren zwischen 1984 und 1997 zusammen.

- Die Nachfrage nach der steuerlich begünstigten Pflegezusatzversicherung („Bahr-Pflege“) boomt. Ende Dezember 2013 gab es bereits 350.100 (laufende) Verträge. Damit sind innerhalb von 12 Monaten mehr staatlich geförderte Pflegezusatzversicherungen abgeschlossen worden als im Bereich der klassischen (steuerlich ungeförderten) Pflegeversicherung in den ersten 10 Jahren zwischen 1984 und 1994 zusammen.

- Nicht nur bei Einführung der Pflegeversicherung hat die PKV ihre Innovationsstärke bewiesen. Auch im Bereich der Pflegequalität hat die PKV neue Vorbilder geschaffen. Das Spektrum reicht von der Pflegeberatung (COMPASS) über Qualitätsprüfungen in Pflegeheimen und bei Pflegediensten („Pflege-TÜV“) bis hin zur wissenschaftsbasierten Verbesserung der Versorgungspraxis (ZQP).

Die Menschen erkennen zusehends den Bedarf einer zusätzlichen Vorsorge, weil die Pflegepflichtversicherung immer nur einen ,Teilkasko'-Schutz bieten kann. Und für kaum ein Risiko kann man - ergänzend zur Pflegepflichtversicherung finanziell so gut und langfristig vorsorgen wie für die Pflege, die meist erst im hohen Alter eintritt. Und trotzdem hinken die tatsächlichen Abschlüsse im Bereich der Pflegezusatzversicherung noch weit hinter dem Bedarf hinterher. Das Pflegerisiko droht - ohne weitere Aufklärung - weiter verdrängt zu werden.

Die (ergänzende) Vorsorge in der Pflege muss zwingend kapitalgedeckt sein. Denn der überwiegende Teil der Wissenschaft war und ist der Meinung, dass sich gerade das Pflegerisiko für das Kapitaldeckungsverfahren eignet. Angesichts der Nachteile der Umlagefinanzierung im demografischen Wandel und angesichts des Tatbestandes, dass das Pflegerisiko noch mehr als das Krankheitsrisiko ein altersabhängiges, im höheren Alter exponentiell ansteigendes Risiko darstellt, hat die Private Krankenversicherung (PKV) auch in der Pflegeversicherung von Anfang an (seit 1984) auf das Prinzip der Kapitaldeckung gesetzt.

Das Umlageverfahren dagegen muss im demographischen Wandel mehr denn je infrage gestellt werden. Umso bedauerlicher ist es, dass die Bundesregierung im Koalitionsvertrag ein Zeichen in die falsche Richtung setzt. Die geplante Anhebung des Beitragssatzes der Sozialen Pflegeversicherung (SPV) weitet das Umlagesystem aus. Die Pläne zum Aufbau eines ,Pflegevorsorgefonds‘ spiegeln zwar die richtige Erkenntnis, dass die Umlagefinanzierung der Pflegeversicherung nicht zukunftsfest ist, sodass mehr finanzielle Vorsorge der heute aktiven Jahrgänge nötig ist. Aber das von den Koalitionsparteien gewählte Instrument ist falsch.

Der Konstruktionsfehler besteht darin, dass eine staatliche Kapitalreserve niemals sicher ist vor der Gefahr einer Zweckentfremdung. Daran ändert auch die geplante Verwaltung bei der Bun-

31 Vertreten von Wolfgang Vogt MdB, Parlamentarischer Staatssekretär im Bundesministerium für Arbeit. 
desbank nichts. Das beweisen schon die aktuellen Koalitionspläne zu Lasten der Reserven der Rentenversicherung. Nur privatrechtlich garantierte Eigentumsan- sprüche können eine langfristige Vorsorge sichern. Dies beweist eindrucksvoll der inzwischen auf mehr als 25,7 Milliarden Euro gewachsene Kapitalstock der Privaten Pflegeversicherung. Er hat sich in den letzten zehn Jahren mehr als verdoppelt - zweckgebunden zu Gunsten der Versicherten.

\title{
Literatur
}

Besche, A. (1995), Die Pflegeversicherung, 1. Auflage, Bonn.

Bundesrat (1986), Drucksache 81/86 vom 7.2.1986, „Entwurf eines Gesetzes zur Absicherung des Risikos der Pflegebedürftigkeit (Pflegeversicherungsgesetz)."

Deutscher Bundestag (1982/1983), Bundestagdrucksachen $9 / 2046$ und $9 / 2401$ vom 22.10.1982/28.01.1983.

Frankfurter Allgemeine Zeitung (2013), Pflege-Bahr stark nachgefragt, 14.11.2013. Hallesche Krankenversicherung (1984), Geschäftsbericht.

Hallesche Krankenversicherung (1985), Geschäftsbericht.

Holl, A.; Kakies. P.; Richter, A. (1985), Die Ableitung der Pflegewahrscheinlichkeiten für den Mustergeschäftsplan der Pflegerentenversicherung, in: Blätter der Deutschen Gesellschaft für Versicherungs- und Finanzmathematik, Oktober 1985, Volume 17., S. $163-178$.
Jung, K.; Schweitzer, R. (1995), Die neue Pflegeversicherung, Sozialgesetzbuch XI, Bonn.

Koch, P.; Uleer, C. (1997), Entwicklungslinien eines Versicherungszweiges von den Anfängen bis zur Gegenwart, Köln

Kuratorium Deutsche Altershilfe (1974),

Gutachten über die stationäre Behandlung von Krankheiten im Alter und über die Kostenübernahme durch die gesetzlichen Krankenkassen.

Münchener Verein Krankenversicherung (o.J.), Tarifbedingungen Tarif 400 Pflegetagegeldversicherung.

Riedel, H. (2003), Private Compulsory Longterm Care Insurance in Germany, in: The Geneva Papers on Risk and Insurance, Vol. 28, No. 2, S. 275-293.

Statistisches Bundesamt (2011), Demografischer Wandel in Deutschland - Bevölkerungsund Haushaltsentwicklung im Bund und in den Ländern, Heft 1, Ausgabe 2011, Wiesbaden.

Statistisches Bundesamt (2012), Durchschnittliche weitere Lebenserwartung nach
Altersstufen ab 1871, www.destatis.de, Wiesbaden

Verband der Privaten Krankenversicherung (1981), Die private Krankenversicherung im Jahre 1980, Rechenschaftsbericht, Köln.

Verband der Privaten Krankenversicherung (1982), Die private Krankenversicherung im Jahre 1981, Rechenschaftsbericht, Köln.

Verband der Privaten Krankenversicherung (1984), Die private Krankenversicherung im Jahre 1983, Rechenschaftsbericht, Köln.

Verband der Privaten Krankenversicherung (1987), Die private Krankenversicherung im Jahre 1986, Rechenschaftsbericht, Köln.

Verband der Privaten Krankenversicherung (1989), Die private Krankenversicherung im Jahre 1988, Rechenschaftsbericht, Köln.

Verband der Privaten Krankenversicherung (1990), Die private Krankenversicherung im Jahre 1989, Rechenschaftsbericht, Köln

WELT (2013), Pflege-Bahr ist Verkaufsschlager, 16.11.2013.

\section{Die Zwangsbehandlung auf dem Prüfstand}

Voraussetzungen der Zwangsbehandlung und die Auswirkungen der jüngsten Gerichtsbeschlüsse auf die Praxis

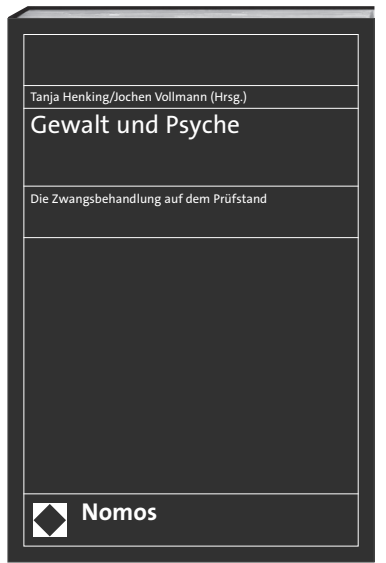

\author{
Gewalt und Psyche \\ Die Zwangsbehandlung auf dem Prüfstand
}

Herausgegeben von Tanja Henking und Jochen Vollmann

2014, 236 S., brosch., 52,-€

ISBN 978-3-8487-0281-7

www.nomos-shop.de/20657

Auf der interdisziplinären Tagung des Instituts für Medizinische Ethik und Geschichte der Medizin der Ruhr-Universität Bochum diskutierten Psychiater, Medizinethiker und Juristen die Voraussetzungen der Zwangsbehandlung und die Auswirkungen der Gerichtsbeschlüsse auf die Praxis. Sie reflektierten die Anforderungen an ein neues Gesetz und hinterfragten die medizinische Indikation der Zwangsbehandlung. 